\title{
EVALUACIÓN DE UNA PRUEBA DE ELISA CON ANTÍGENO METABÓLICO DE Fasciola hepatica PARA EL DIAGNÓSTICO DE FASCIOLOSIS HUMANA EN CAJAMARCA, PERÚ*
}

\author{
Hernán Cornejo ${ }^{1, a}$, Fátima Oblitas ${ }^{2, b}$, Sandro Cruzado ${ }^{2, b}$, William Quispe 3 ,a
}

\begin{abstract}
RESUMEN
Se obtuvo el antígeno metabólico (antígeno excreción - secreción) de Fasciola hepatica de ovinos infectados de Cajamarca, con una concentración proteica de $1005 \mu \mathrm{g} / \mu \mathrm{L}$, compuesta principalmente por proteínas de peso molecular entre 1,2 y 170 KDa. Se detectaron bandas de 170; 150; 31; 24; 18-14 y 10 kDa. Con este antígeno se desarrolló una prueba de ELISA y se determinó su punto de corte en 0,140 . Se evaluó 33 sueros de pacientes con fasciolosis confirmada por visualización de huevos en heces, 177 sueros de pacientes sin fasciolosis provenientes de áreas endémicas de Cajamarca y 88 sueros de pacientes con otras infecciones parasitarias y bacterianas. Se encontró una sensibilidad de 97,0\%, especificidad de 96,6\%, valor predictivo positivo de $78,1 \%$ y valor predictivo negativo de $99,6 \%$. Se encontró reacción cruzada en $9 / 88$ sueros evaluados. Se recomienda la implementación y uso de esta prueba para el diagnóstico de fasciolosis.
\end{abstract}

Palabras clave: Fascioliasis/diagnóstico; Fasciola hepatica; Pruebas inmunológicas; ELISA, Sensibilidad y especificidad; Perú (fuente: DeCS BIREME).

\section{EVALUATION OF AN ELISA TEST WITH Fasciola hepatica METABOLIC ANTIGEN FOR DIAGNOSIS OF HUMAN FASCIOLIASIS IN CAJAMARCA, PERU}

\begin{abstract}
Metabolic (excretion/secretion) antigen was obtained from sheep infected with Fasciola hepatica, with a $1005 \mu \mathrm{g} / \mu \mathrm{L}$ of protein concentration, composed principally by proteins of molecular weight between 1.2 and $170 \mathrm{KDa}$. Bands of $170,150,31,24,18-14$ and $10 \mathrm{kDa}$ were detected. With this antigen an ELISA test was developed and the cut off was determined in 0.140 . We evaluated 33 serums of patient with fascioliasis confirmed by visualization of eggs in feces, 177 serums of persons without fascioliasis from endemic rural areas of Cajamarca and 88 serums of patients with others parasitic and bacterial infections. We found a $97.0 \%$ of sensitivity, 96.6 specificity, $78.1 \%$ predictive positive value, 99.6 $\%$ predictive negative value. In 9/88 serums was found cross reactions. We recommended the implementation and use of this test for the fascioliasis diagnosis.
\end{abstract}

Key words: Fascioliasis/diagnosis; Fasciola hepatica; Immunologic test; Sensitivity and specificity; Peru (source: MeSH NLM).

\section{INTRODUCCIÓN}

La fasciolosis es una zoonosis parasitaria causada por un tremátodo denominado Fasciola hepática, se estima que existen entre 2,6 y 17 millones de personas infectadas en el mundo ${ }^{(1)}$ y es endémica en comunidades rurales del Perú ${ }^{(2,3)}$.
Cajamarca es una de las regiones andinas peruanas endémicas con alta prevalencia de fasciolosis, sobre todo en población rural, las prevalencias humanas se encuentran entre $6,3 \%$ y $47,7 \%$, siendo los niños de edad escolar los más afectados. La prevalencia se mantiene alta por la falta de educación sanitaria y escasos recursos económicos de los pobladores que

\footnotetext{
Laboratorio de Inmunoserologia, Laboratorio de Referencia Regional, Dirección Regional de Salud, Cajamarca, Perú.

Facultad de Farmacia y Bioquímica, Universidad Privada Antonio Guillermo Urrelo, Cajamarca, Perú.

Laboratorio de Zoonosis Parasitaria, Centro Nacional de Salud Pública, Instituto Nacional de Salud, Lima, Perú.

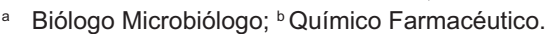

* Este trabajo es un resumen de la tesis para optar el título de químico farmacéutico: Cruzado S, Oblitas F. Obtención de antígeno metabólico de Fasciola hepatica e implementación de la técnica ELISA para el diagnóstico de fasciolasis humana Cajamarca - 2009. Cajamarca: Universidad Privada Antonio Guillermo Urrelo; 2009.
}

Recibido: 15-09-10 Aprobado: 24-11-10 
dejan deambular libremente a los animales herbívoros en zonas cercanas a fuentes de agua corriente, incrementando el riesgo de infectarse por consumir berros $u$ otros vegetales de tallo corto con quistes de metacercarias (forma infectante) ${ }^{(4-7)}$.

La fasciolosis humana tiene tres estadios clásicos: agudo, prepatente y crónico, en el estadio agudo no encontramos huevos de $F$. hepatica en las heces, pues el parásito está migrando y las pruebas serológicas son las que ayudan a detectar la infección. En la fase prepatente y crónica, el trematodo ya alcanzó las vías biliares, y la serología y los exámenes de heces son positivos; sin embargo, la excreción de los huevos es intermitente; por ello, el inmunodiagnóstico es el método de elección para el diagnóstico de fasciolosis pues detecta fase aguda, prepatente y crónica ${ }^{(1,5,8)}$.

Dentro de los métodos más confiables de inmunodiagnóstico, debido a su fácil manejo, alta sensibilidad y especificidad, se encuentran las pruebas inmunoenzimáticas; la más importante es la técnica de ELISA (Enzime-linked immunoabsorbent assay), la cual es utilizada para la detección de anticuerpos y antígenos (ELISA indirecto) ${ }^{(1,9,10)}$. La sensibilidad y especificidad son superiores cuando se trabaja con antígeno de F.hepatica de excreción - secreción (AFHES) que cuando se trabaja con antígeno somático de F. hepatica ${ }^{(11)}$.

A pesar de ser Cajamarca una zona endémica de fasciolosis, no se tiene implementado, para el uso en los servicios de salud, una prueba de inmunoenzimática para el diagnóstico de fasciolosis, por ello, el objetivo del estudio es desarrollar una prueba de ELISA a partir del antígeno metabólico de F. hepatica (AFHES) y evaluar su eficiencia diagnóstica para su implementación.

\section{EL ESTUDIO}

Se desarrolló una prueba de ELISA para el diagnóstico de fasciolosis humana a partir de un antígeno metabólico obtenido de parásitos locales y se evaluó su eficiencia diagnóstica en población rural de zonas endémicas de Cajamarca entre julio de 2008 y febrero de 2009. El estudio fue revisado y aprobado por el Comité de Investigación de la Dirección Regional de Salud Cajamarca, todos los participantes del estudio firmaron el consentimiento y asentimiento informado, según correspondía. Los resultados de las pruebas fueron remitidos a los establecimientos de salud a los que pertenecían los participantes y quienes, en caso de ser positivos, recibirán tratamiento con triclabendazol.

\section{OBTENCIÓN DEL ANTÍGENO METABÓLICO DE FASCIOLA}

Se obtuvo hígados de ovino infectados con F. hepatica en el camal municipal de Cajamarca. En el laboratorio se realizó cortes transversales y se presionó ligeramente los canalículos biliares para sacar a los tremátodos, luego con ayuda de una pinza se les extrajo sin dañarlas, se llevaron a un frasco con PBS pH 7,2 en un volumen de $300 \mathrm{~mL}$. Se lavó dos veces con PBS pH 7,2 rápidamente hasta que estén limpias y eliminen todos los restos de sangre y bilis del hospedador.

Se colocó 200 ejemplares de F. hepatica en una placa petri estéril con $100 \mathrm{~mL}$ de PBS $(\mathrm{pH} 7,2)$. Se incubó a $37^{\circ} \mathrm{C}$ por cuatro horas luego se refrigeró a $4{ }^{\circ} \mathrm{C}$ durante toda la noche. Se retiró las fasciolas y se recogió el PBS (antígeno de excreción - secreción), el cual se centrifugó a $10000 \mathrm{rpm}$ durante una hora a $4{ }^{\circ} \mathrm{C}$. Finalmente, se distribuyó proporcionalmente el sobrenadante en crioviales y se congeló a -20 o $-80{ }^{\circ} \mathrm{C}$.

Para determinar la concentración de proteínas, se usó el método de Lowry ${ }^{(9,10)}$. Se construyó una curva patrón a partir de una solución patrón (BSA) $(1 \mathrm{mg} / \mathrm{mL})$ y la concentración de proteínas del antígeno de secreción excreción de Fasciola hepatica, se determinó por interpolación de los valores de absorbancia en la curva patrón. Para la caracterización de los pesos moleculares de las proteínas antigénicas se realizó la electroforesis en chip ${ }^{(12,13)}$.

Los antígenos (AFHES) obtenidos tuvieron una concentración proteica de $1005 \mu \mathrm{g} / \mu \mathrm{L}$ y están compuestos principalmente por proteínas de peso molecular entre 1,2 a $170 \mathrm{kDa}$, se detectó bandas de 170; 150; 31; 24; 18-14 y $10 \mathrm{kDa}$ de peso molecular por electroforesis en chip (Figura 1).

El antígeno (AFHES) obtenido de ganado ovino de Cajamarca fue comparado con los antígenos (AFHES) de ganado ovino proporcionados por el Instituto Nacional de Salud (INS) y de ganado bovino de la Unidad de Parasitología de la Facultad de Veterinaria de Lugo (Universidad de Santiago de Compostela, España); donde se observó una composición común de proteínas que van desde 10 - $50 \mathrm{kDa}$ en los tres preparados antigénicos (Figura 2).

\section{ELABORACIÓN DE LA PRUEBA ELISA}

El antígeno obtenido (AFHES-Cajamarca) se impregnó en placas de microtitulación (100 $\mu \mathrm{L} /$ pocillo) diluido en Tris- $\mathrm{HCl} 0,01 \mathrm{M}, \mathrm{pH}$ 7,5 a una concentración de 1 $\mu \mathrm{g}$ prot/100 $\mu \mathrm{L}$, se cubrió las placas con parafilm y se 


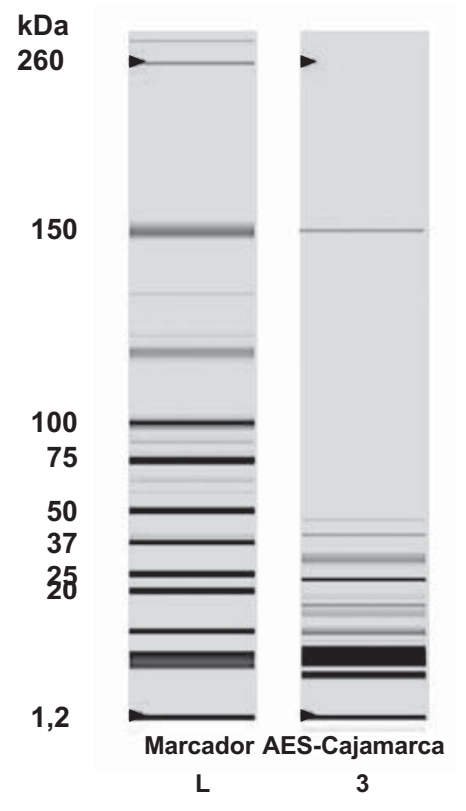

Figura 1. Caracterización por electroforesis en Chip del antígeno metabólico de excreción-secreción de Fasciola hepatica (AFHES) de Cajamarca.

dejó toda la noche en refrigeración. Al día siguiente se realizó el lavado con PBS-Tween 20 (200 $\mu$ l por pozo) en cinco oportunidades para quitar el exceso de antígeno, posteriormente, se adicionó a cada pocillo $200 \mu \mathrm{lde}$ solución de bloqueo (PBS-Tween20-Leche5\%) e incubó a $37{ }^{\circ} \mathrm{C}$ por 30 minutos. Luego se realizó un nuevo lavado (200 $\mu \mathrm{l} /$ pozo) por cinco veces; de esta manera, la placa impregnada y bloqueada quedó lista para iniciar la corrida ELISA.

\section{EVALUACIÓN DE LA PRUEBA DE ELISA}

Para obtener sueros positivos y negativos a Fasciola hepatica se recolectó muestras de sangre venosa en población general y escolares de los distritos de Chetilla, Jesús, La Encañada, Llacanora, Namora y Matara ubicados en la provincia de Cajamarca y el distrito de Catilluc en la provincia de San Miguel, todos ellos son endémicos para fasciolosis. La selección de los sujetos fue por conveniencia y coordinada con el personal de los establecimientos de salud de cada comunidad, se seleccionó a las escuelas con mayor población estudiantil tanto del nivel primario como secundario y se incluyó a todos los que desearon participar; en forma similar se procedió con población adulta en la comunidad quienes tenían más de tres meses de residencia en la zona de estudio. A cada persona se le extrajo $5 \mathrm{~mL}$ de sangre venosa y se le solicitó una muestra de heces que fue procesada por la técnica de sedimentación rápida

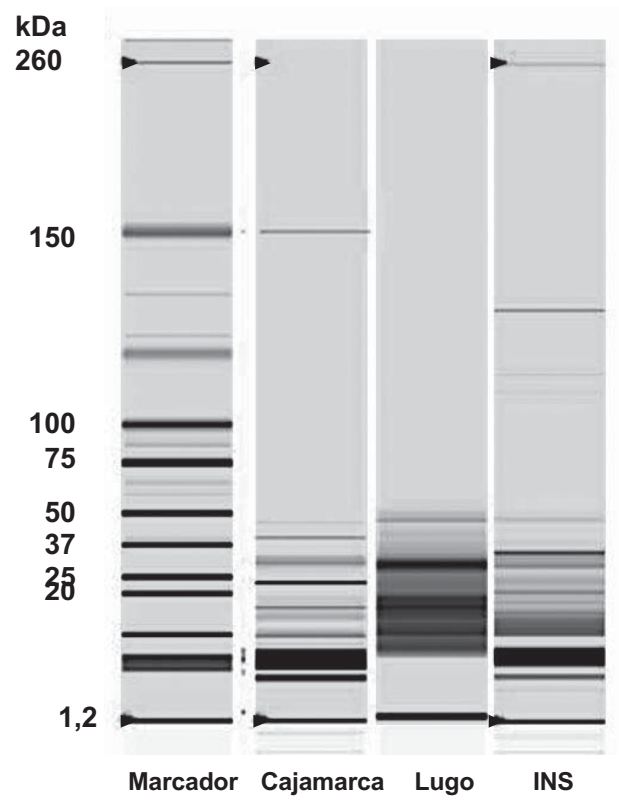

Figura 2. Comparación y caracterización por electroforesis de antígenos (AFHES) Cajamarca, Lima (INS) y Lugo (España).

(TSR) ${ }^{(14)}$ en el Laboratorio de Referencia Regional de Cajamarca.

Se extrajo 210 muestras de sangre, de ellas se obtuvo el suero por centrifugación a $2500 \mathrm{rpm}$ durante cinco minutos, luego se separó en alícuotas de 1,5 mL y se conservó a $-20{ }^{\circ} \mathrm{C}$ hasta su procesamiento en el Laboratorio de Zoonosis Parasitaria del Instituto Nacional de Salud en Lima, Perú.

Se estableció el punto de corte usando el promedio de los títulos obtenidos de los sueros de personas que tuvieron el examen coproparasitológico negativo (177 personas) más tres veces la desviación estándar, que fue de 0,140 D.O. a una longitud de onda de $490 \mathrm{~nm}$; tomando en cuenta este valor, se consideró a los sueros de los pacientes como positivos o negativos.

Para la evaluación de la prueba de ELISA se usó tres tipos de sueros: 33 sueros positivos positivos a fasciolosis diagnóstico por la TSR en heces, 177 sueros negativos por la prueba de TSR y 88 sueros positivos a otras enfermedades bacterianas y parasitarias pero negativos a fasciolosis por western blot provenientes de la seroteca del Instituto Nacional de Salud para evaluar reacciones cruzadas, estos sueros fueron positivos a hidatidosis (9), cisticercosis (10), toxoplasmosis (5), leptospirosis (2), hymenolepiosis (3), giardiosis (21), ascariosis (6), estrongiloidiosis (5), blastocistosis (15), 


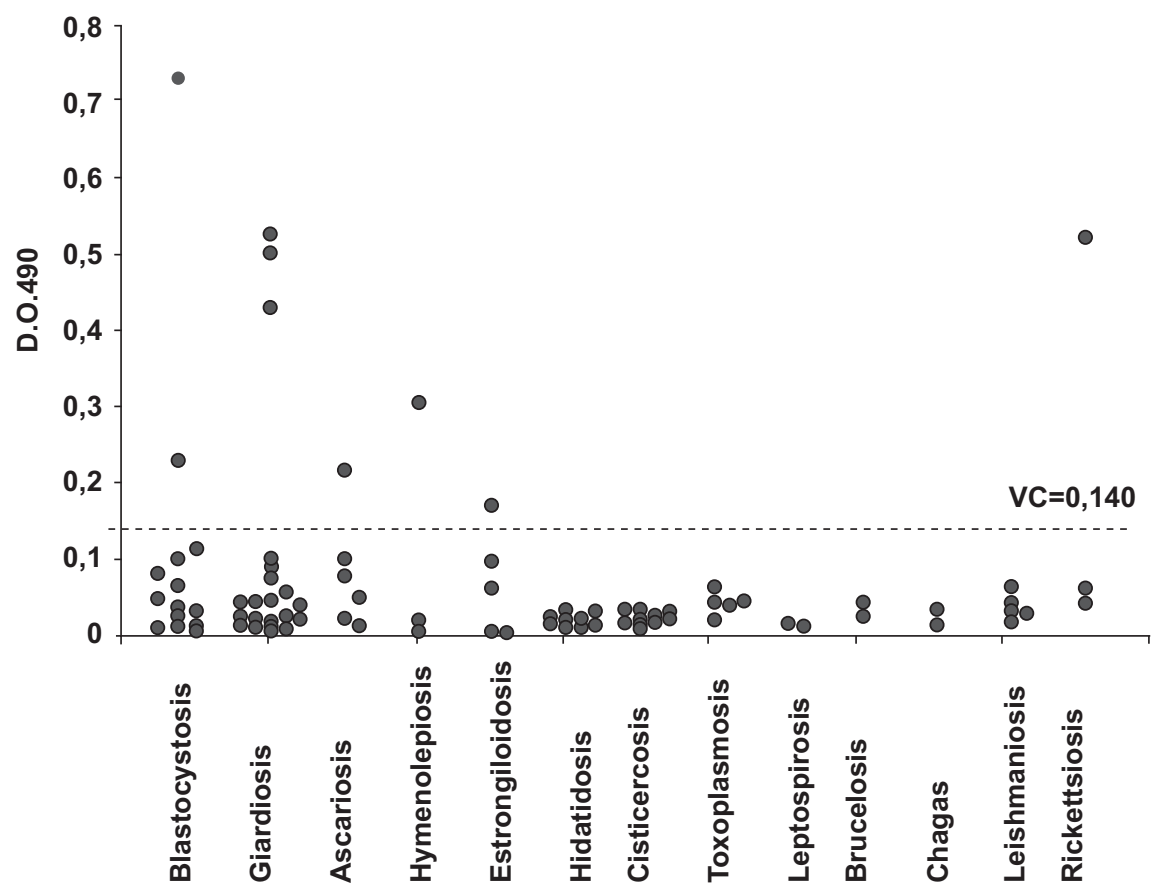

Figura 3. Distribución de los sueros positivos a otras etiologías según los valores obtenidos con la prueba ELISA con el antígeno metabólico de F. hepatica.

Brucelosis (2), enfermedad de Chagas (2), Leishmaniosis (5) y Rickettsiosis (3).

Los resultados fueron procesados con el programa Epidat v3.1, se calculó los valores de sensibilidad, especificidad y valores predictos positivos (VPP) y negativos (VPN), así como las razones razones de verosimilitud positiva (RVP) y negativa (RVN).

Delaspersonascondiagnósticoconfirmadodefasciolosis, una tuvo resultado negativo por ELISA (1/33), de las personas con sueros positivos a otras enfermedades 9/88 fueron positivos (Tabla 1), la evaluación según tipo de suero positivo a otras infecciones y el resultado del ELISA se observa en la Figura 3.

Tabla 1. Evaluación del ELISA con el antígeno metabólico de F. hepatica en el diagnóstico de fasciolosis humana.

\begin{tabular}{lccc}
\hline \multirow{2}{*}{$\begin{array}{c}\text { ELISA antígeno } \\
\text { metabólico de } \boldsymbol{F} .\end{array}$} & \multicolumn{2}{c}{ Fasciolosis } & Total \\
\cline { 2 - 3 } hepatica & Positivos $^{*}$ & Negativost & \\
\hline Positivo & 32 & 9 & 41 \\
Negativo & 1 & 256 & 257 \\
Total & 33 & 265 & 298 \\
\hline
\end{tabular}

* Los casos positivos fueron diagnosticados por la visualización de huevos de $F$. hepatica por la técnica de sedimentación rápida (TSR) en heces.

$\dagger 177$ casos negativos tuvieron una prueba negativa en el coproparasitológico y 88 provinieron de sueros positivos a otras infecciones pero negativos a $F$. hepatica por western blot.
La sensibilidad de la prueba fue de 97,0\% (IC95\%: 89,6100\%), especificidad de 96,6\% (IC95\%: 94,2-98,9\%), VPP de 78,1\% (IC95\%: 64,2-91,9\%), VPN de 99,6\% (IC95\%: 98,7-100\%), con RVP de 28,6 (IC95\%: 15,0$54,4)$ y RVN de 0,03 (IC95\%: 0,00-0,22).

\section{DISCUSIÓN}

Diversos estudios indican que la mayor respuesta inmune frente al antígeno de Fasciola hepatica, se da con proteínas entre 12,4 a $60 \mathrm{kDa}{ }^{(12,13)}$, el antígeno de Fasciola hepatica de Cajamarca cuenta con pesos moleculares de 170; 150; 31; 24; 18-14 y 10 kDa de los cuales, 24, 18-14 kDa están dentro del rango con mayor respuesta antigénica.

La proteína con peso molecular de 24 kDa es producto de excreción - secreción, propia del tegumento del parásito que parece formar parte de las células tegumentales y gránulos tipo 1 y tipo 2 (T1 y T2) presentes en los estadios inmaduros y de adultos del trematodo ${ }^{(15,16)}$, lo que indica que esta proteína es una de las más importantes en la respuesta inmune.

La prueba de ELISA desarrollada a partir del antígeno metabólico de $F$. hepatica ha demostrado mejor sensibilidad que otras pruebas inmunológicas ensayadas en el Perú, como el FAS2 y FAS1-ELISA, western blot y arco2 ${ }^{(5,10,14)}$; si bien tuvo una buena especificidad esta fue menor que la obtenida por Cordova et al. ${ }^{(10)} \mathrm{con}$ el 
Tabla 2. Evaluación de pruebas inmunológicas para el diagnóstico de fasciolosis humana en el Perú.

\begin{tabular}{|c|c|c|c|c|c|c|c|}
\hline Autor & Prueba & $\begin{array}{c}\text { Sueros } \\
\text { evaluados }\end{array}$ & Lugar - año & s & $E$ & VPP & VPN \\
\hline Cornejo & ELISA-AFHES & $298(\mathrm{~N}, \mathrm{P}, \mathrm{O})^{*}$ & Cajamarca -2009 & $97,0 \%$ & $96,6 \%$ & $78,1 \%$ & $99,6 \%$ \\
\hline Maco (14) & ELISA-FAS2 & $145(\mathrm{~N}, \mathrm{P})$ & Junín - 2000 & $96,7 \%$ & $91,2 \%$ & $75,0 \%$ & $99,1 \%$ \\
\hline Espinoza ${ }^{(5)}$ & ELISA-FAS2 & $237(N, P)$ & Cajamarca - 2001 & $96,5 \%$ & $92,2 \%$ & $79,7 \%$ & $98,8 \%$ \\
\hline Cordova ${ }^{(10)}$ & ELISA-FAS2 & $138(\mathrm{~N}, \mathrm{P}, \mathrm{O}) \dagger$ & Lima - 1997 & $94,7 \%$ & $100 \%$ & $100 \%$ & $98,6 \%$ \\
\hline Espinoza ${ }^{(5)}$ & ELISA-FAS2 & $158(N, P)$ & Junín - 2000 & $92,6 \%$ & $89,6 \%$ & $69,4 \%$ & $97,9 \%$ \\
\hline Cordova ${ }^{(10)}$ & ELISA-FAS1 & $138(\mathrm{~N}, \mathrm{P}, \mathrm{O}) \dagger$ & Lima - 1997 & $89,4 \%$ & $98,0 \%$ & $94,5 \%$ & $96,2 \%$ \\
\hline Espinoza ${ }^{(5)}$ & ELISA-FAS2 & $231(N, P)$ & Puno - 2001 & $88,3 \%$ & $70,8 \%$ & $51,5 \%$ & $94,5 \%$ \\
\hline Maco ${ }^{(14)}$ & Western blot & $144(\mathrm{~N}, \mathrm{P})$ & Junín - 2000 & $71,9 \%$ & $93,8 \%$ & $76,7 \%$ & $92,1 \%$ \\
\hline Maco ${ }^{(14)}$ & Arco2 & $143(N, P)$ & Junín - 2000 & $34,5 \%$ & $98,3 \%$ & $83,3 \%$ & $85,5 \%$ \\
\hline
\end{tabular}

* Incluyó 88 sueros de pacientes con otras infecciones parasitarias y bacterianas.

† Incluyó 54 sueros de pacientes con otras parasitosis.

FAS2-ELISA. Lo que indica que esta prueba es útil para el diagnóstico de fasciolosis.

Una limitación del estudio, es que la visualización de huevos de Fasciola hepatica en heces, como prueba utilizada como gold standar, solo puede detectar los casos de fasciolosis en estadio crónico o prepatente, por lo que no se puede conocer el rendimiento de la prueba en estadios agudo; sin embargo, esta limitación es compartida con todos los estudios previos realizados y con los que comparamos nuestros resultados.

Encontramos reacciones cruzadas con sueros de pacientes con blastocitosis (2/15), giardiosis (1/20), ascariosis (1/3), estrongiloidosis (1/6) y Rickettsiosis (1/3), a diferencia del estudio de Cordova et al.(10) que encontró que el FAS2-ELISA no tuvo reacciones cruzadas con sueros de pacientes con enfermedades parasitarias a diferencia de lo que encontramos, que podría deberse al nivel de purificación que tiene el antígeno que ellos usaron. Futuros estudios podrían trabajar en purificar mejor los antígenos de excreción secreción que usamos y evaluar las reacciones cruzadas que encontramos, así mismo tener la certeza de que los sueros trabajados provengan de pacientes con la parasitosis a estudiar y que no hayan tenido fasciolosis, ya que un problema de estas pruebas inmunológicas es que no distinguen entre infección actual y pasada. Además, estos sueros fueron western blot negativos, que tiene una menor sensibilidad que el ELISA y en los casos de enfermedades bacterianas no se tiene la seguridad de un examen coproparasitológico negativo, como es el caso de reacción cruzada por Rickettsiosis.

El rendimiento de la prueba desarrollada en el Laboratorio de Referencia Regional de Cajamarca señala su idoneidad para el diagnóstico de fasciolosis humana, por lo que se recomienda su implementación y uso.

\section{Financiamiento}

Esta investigación fue autofinanciada, contando con el apoyo técnico del Laboratorio de Referencia Regional Cajamarca e Instituto Nacional de Salud.

\section{Conflictos de Interés}

Los autores declaran no tener conflictos de interés en la publicación de este artículo.

\section{REFERENCIAS BIBLIOGRÁFICAS}

1. Mas-Coma MS, Esteban JG, Bargues MD. Epidemiology of human fascioliasis: a review and proposed new classification. Bull World Health Organ. 1999;77(4):340-46.

2. Esteban J, Gonzales C, Bargues M, Angles R, Sánchez C, Naquira C, et al. High fascioliasis infection in children linked to a man-made irrigation zone in Peru. Trop Med Int Health. 2002;7(4):339-48.

3. Marcos LA, Terashima A, Leguia G, Canales M, Espinoza JR, Gotuzzo E. La infección por Fasciola hepatica en el Perú: una enfermedad emergente. Rev Gastroenterol Peru.2007;27(4):389-96.

4. Albán M, Jave J, Quispe T. Fasciolasis en Cajamarca. Rev Gastroenterol Peru. 2002;22(1):28-32.

5. Espinoza JR, Maco V, Marcos L, Saez S, Neyra V, Terashima A, et al. Evaluation of Fas2-ELISA for the serological detection of Fasciola hepatica infection in humans. Am J Trop Med Hyg. 2007;76(5):977-82.

6. Ortiz P, Cabrera M, Jave J, Claxton JR, Williams D. Human fasciolasis: prevalence and treatment in a rural area of Peru. Infect Dis Rev. 2000;2(1):42-46.

7. Raunelli F, Gonzalez S. Strategic control and prevalence of Fasciola hepatica en Cajamarca, Peru. A pilot study. Intern J Appli Res Vet Med. 2009;7(4):145-52. 
8. Rivera Marrero CA, Santiago N, Hillyer GV. Evaluation of immunodiagnostic antigens in the excretory-secretory products of Fasciola hepatica. J Parasitol. 1988;74(4):646-52.

9. Cordova M, Herrera P, Nopo L, Bellatin J, Naquira C, Guerra $\mathbf{H}$, et al. Fasciola hepatica cysteine proteinases: inmunodominant antigens in human fasciolosis. Am J Trop Med Hyg. 1997;57(6):660-66.

10. Cordova M, Reategui L, Espinoza JR. Immunodiagnosis of human fascioliasis with Fasciola hepatica cysteine proteinases. Trans R Soc Trop Med Hyg. 1999;93(1):54-57.

11. Incil E. Reconocimiento antigénico de productos de Fasciola hepatica en infecciones humanas. [Tesis de Bachiller] Cajamarca: Facultad de Ciencias Veterinarias, Universidad Nacional de Cajamarca; 2000.

12. Espino AM, Dumenigo BE, Fernández R, Finlay CM. Immunodiagnosis of human fascioliasis by enzyme-linked immunosorbent assay using excretorysecretory products. Am J Trop Med Hyg. 1997;37(3):605-8.

13. Fredes $F$, Alarcón J, llabaca $P$, Alcaíno H. Evaluación diagnóstica de dos proteínas purificadas de Fasciola hepatica mediante ELISA en la fasciolosis ovina. Parasitol Latinoam. 2003;58(3-4):148-51.
14. Maco V, Marcos L, Terashima A, Samalvides F, Miranda E, Espinoza J, et al. Fas2-ELISA y la técnica de sedimentación rápida modificada por Lumbreras en el diagnóstico de la infección por Fasciola hepatica. Rev Gastroenterol Peru. 2002;13(2):49-57.

15. Miranda M, García Z. Aislamiento e identificación in situ de antígenos de Fasciola hepatica. Vet Mex. 1994;25(3):267- 71.

16. Knobloch J. Human fascioliasis in Cajamarca/Peru. II. Humoral antibody response and antigenaemia. Trop Med Parasitol. 1985;36(2):91-93.

Correspondencia: Blgo. Hernán Daniel Cornejo Pacherres. Dirección: Av Mario Urteaga 500, Cajamarca, Perú.

Telefono: (51-076) 363-864 anexo: 134

Correo electrónico: dcornejop68@yahoo.es

\section{Consulte la versión electrónica de la Revista Peruana de Medicina Experimental y Salud Pública en www.pubmed.gov}

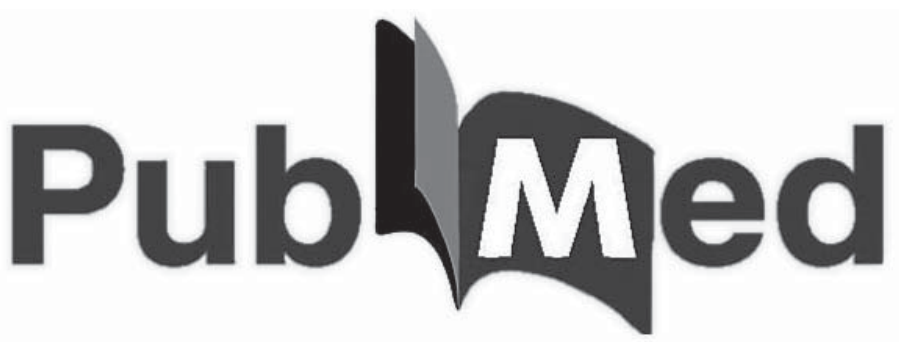

